\title{
Ffar2 expression regulates leukaemic cell growth in vivo
}

Laure B Bindels ${ }^{1}$, Paolo E Porporato ${ }^{2,3}$, Sarah Ducastel ${ }^{4}$, Martina Sboarina ${ }^{2}$, Audrey M Neyrinck ${ }^{1}$, Evelyne M Dewulf ${ }^{1}$, Olivier Feron ${ }^{2}$, Sophie Lestavel ${ }^{4}$, Patrice D Cani ${ }^{1,5}$, Bart Staels ${ }^{4}$, Pierre Sonveaux ${ }^{2}$ and Nathalie M Delzenne ${ }^{*, 1}$

${ }^{1}$ Metabolism and Nutrition Research Group, Louvain Drug Research Institute, Université Catholique de Louvain, Avenue Mounier 73, Box B1.73.11, Brussels 1200, Belgium; ${ }^{2}$ Pole of Pharmacology, Institut de Recherche Expérimentale et Clinique (IREC), Université Catholique de Louvain, Avenue Mounier 52, Box B1.53.09, Brussels 1200, Belgium; ${ }^{3}$ Department of Molecular Biotechnology and Health Sciences, Molecular Biotechnology Center, University of Torino, Via Nizza 52, Torino 10126, Italy; ${ }^{4}$ European Genomic Institute for Diabetes (EGID), Université Lille, INSERM UMR 1011, Institut Pasteur de Lille, CHU de Lille, Rue du Professeur Calmette 1, Lille F-59019, France and ${ }^{5}$ Walloon Excellence in Life Sciences and Biotechnology (WELBIO), Louvain Drug Research Institute, UCL, Brussels B-1200, Belgium

Background: Activation of free fatty acid receptor 2 (FFAR2) by microbiota-derived metabolites (e.g., propionate) reduces leukaemic cell proliferation in vitro. This study aims to test whether Ffar2 expression per se also influences leukaemia cell growth in vivo.

Methods: Bcr-Abl-expressing BaF cells were used as a leukaemia model and the role of Ffar2 was evaluated in Balb/c mice after lentiviral shRNA transduction.

Results: Our data formally establish that reduced leukaemic cell proliferation is associated with increased Ffar2 expression in vivo and in vitro. Going beyond association, we point out that decreasing Ffar2 expression fosters cancer cell growth in vitro and in vivo.

Conclusions: Our data demonstrate the role of Ffar2 in the control of leukaemic cell proliferation in vivo and indicate that a modulation of Ffar2 expression through nutritional tools or pharmacological agents may constitute an attractive therapeutic approach to tackle leukaemia progression in humans.

Short-chain fatty acids (SCFA), such as acetate, propionate and butyrate, are microbial byproducts of fermentation that are involved in the gut microbiota-host crosstalk through several pathways (Bindels et al, 2013; Canfora et al, 2015). Free fatty acid receptor 2 (FFAR2), also known as GPR43 or FFA2, is a G-protein coupled receptor that binds propionate. Its activation reduces leukaemic cell proliferation in vitro (Bindels et al, 2013). In a mouse model of leukaemia consisting in the transplantation of BcrAbl-transfected BaF cells, feeding inulin-type fructans (ITF), a non-digestible fermentable fiber changing the gut microbiota, leads to increased portal propionate levels and reduced hepatic leukaemic cell proliferation (Bindels et al, 2012). We and others have documented that beside the canonical histone deacetylase pathway, the FFAR2 pathway could also mediate the anti-proliferative effect of SCFA such as propionate (Tang et al, 2011; Bindels et al, 2012; Shi et al, 2014). While overexpression of Ffar2 induces apoptosis (Tang et al, 2011; Shi et al, 2014), our data reveal that its knocking down increases the growth of leukaemic cells in vivo.

\section{MATERIALS AND METHODS}

Chemicals. All chemicals were purchased from Sigma-Aldrich (St Louis, MO, USA), except CMTB [4-chloro- $\alpha$-(1-methylethyl)$\mathrm{N}$-2-thiazolyl-benzeneacetamide] (Ambinter, Paris, France) and imatinib mesylate (Cayman Chemical, Tallinn, Estonia).

*Correspondence: Professor NM Delzenne; E-mail: nathalie.delzenne@uclouvain.be 
Cell culture. BaF cells transfected with Bcr-Abl were a kind gift of Dr K Bhalla (MCG Cancer Center, Medical College of Georgia, Augusta, GA, USA). Cell maintenance, MTT (thiazolyl blue tetrazolium bromide) cell growth assays and counting of intact BaF cells are described in Bindels et al (2012). U937 and HL-60 cells were maintained in RPMI medium 1640 or Iscove's Modified Dulbecco's Medium, respectively, each medium being supplemented with $10 \%$ foetal bovine serum (PAA clone, PAA, Pasching, Austria) and streptomycin $100 \mu \mathrm{g} \mathrm{ml}^{-1}$ and penicillin $100 \mathrm{IU} \mathrm{ml}^{-1}$ (Gibco, Inchinnan, Scotland). For mRNA analysis, $\mathrm{BaF}$ cells were incubated with chemicals or the corresponding vehicle as indicated in figure legends. After incubation, the cells were harvested, washed with PBS, and frozen in Tripure Isolation Reagent (Roche Diagnostics, Penzberg, Germany). To silence Ffar2 expression, $\mathrm{BaF}$ cells were infected with lentiviral particles containing short hairpin RNA (shRNA) sequences. Briefly, pLKO 0.1 lentiviral vectors expressing control shRNA (Addgene 1864, Cambridge, UK) and FFAR2 shRNA (clone TRCN0000027562; Sigma-Aldrich) were used to generate viral particles with the lentiviral packaging mix (Sigma-Aldrich) according to the manufacturer's instructions. After 24 and $48 \mathrm{~h}$, viral supernatant was harvested, titrated, and used to infect BaF cells. Puromycin selection $\left(10 \mu \mathrm{g} \mathrm{ml}^{-1}\right)$ was applied after lentiviral infection.

Animals. In a first series of experiments, BaF cells $\left(1 \times 10^{6}\right.$ cells in $0.1 \mathrm{ml}$ saline) or an equal volume of saline was injected into the tail vein of anesthetised 5-week-old female $\mathrm{BALB} / \mathrm{c}$ mice (Charles River, France). One day after inoculation, half of the mice that were transplanted with $\mathrm{BaF}$ cells received $0.2 \mathrm{~g}$ per day of ITF (Orafti p95, Beneo-Orafti, Oreye, Belgium) in their drinking water for 12 days. In a second series of experiments, 10-week-old male C57BL6/ $\mathrm{J}$ mice (Charles River, France) received or not $0.2 \mathrm{~g}$ per day of ITF for 4 weeks in the drinking water. In a third series of experiments, BaF-shCT or BaF-shFFAR2 $\left(1 \times 10^{6}\right.$ cells in $0.1 \mathrm{ml}$ saline $)$ or an equal volume of saline was injected into the tail vein of anesthetised 5-week-old female BALB/c mice. Mice were necropsied 13 days after inoculation. White blood cell count was performed on a MS9-3 Hematology Analyzer (Melet Schloesing Laboratoires, Osny, France).

Mice were housed in a $12 \mathrm{~h}$ light/dark cycle. Mice were necropsied under anesthesia. Blood samples and organs were harvested and stored at $-80^{\circ} \mathrm{C}$. Mouse housing and all in vivo experiments were approved by the Ethics Committee of the Université catholique de Louvain (Agreement LA 1230314).

mRNA and western blot analyses. mRNA analyses and western Blot of STAT3 were performed as previously described (Bindels et al, 2016; Cacace et al, 2016). Amplification product size and absence of genomic DNA contamination were checked on agarose gel for a subset of samples (Supplementary Figure S1). Primer sequences are indicated in Supplementary Table S1.
Statistical analysis. Results are expressed as mean \pm s.e.m. Student $t$-test was used to compare two groups and one-way ANOVA with Dunnett's post-tests to compare three or more groups. Two-way ANOVA with Bonferroni post-tests was used to examine the influence of two categorical variables. $P<0.05$ was considered statistically significant (Graph-Pad Prism Software, San Diego, CA, USA).

\section{RESULTS}

We previously showed that increasing portal levels of propionate by the administration of ITF leads to decreased leukaemic cell accumulation in mouse liver, as evidenced by a decreased Bcr-Abl expression (Figure 1A) (Bindels et al, 2012). Interestingly, it was associated with an increased Ffar2 expression in the liver of ITFfed leukaemic mice (Figure 1B). Induction of Ffar2 expression was likely restricted to the remaining $\mathrm{BaF}$ cells. Indeed, we found that Ffar2 is 70-fold more expressed in BaF cells than in the mouse liver (BaF cells: $1.000 \pm 0.059$, liver: $0.015 \pm 0.003, n=3$ ), and the administration of ITF to healthy mice for 4 weeks did not influence hepatic Ffar2 expression (Figure 1C).

We further analysed this phenomenon in vitro in $\mathrm{BaF}$ cells, where propionate simultaneously reduced cell proliferation and increased Ffar 2 expression in a dose-dependent manner (Figure 2A and B). Reduced cell proliferation and increased Ffar2 expression were also observed when mimicking the action of propionate on FFAR2 and histone deacetylases using CMTB, a FFAR2 synthetic agonist (Wang et al, 2010), and trichostatin A, an inhibitor of histone deacetylases (Charrier et al, 2006) (Figure 2C-F). Thus, both pathways could be involved in the modulation of Ffar2 expression by propionate. Imatinib, a tyrosine kinase inhibitor targeting Bcr-Abl (Druker, 2008), also reduced cell growth and increased Ffar2 expression (Figure $2 \mathrm{G}$ and $\mathrm{H}$ ), expanding the concept of an association between cell proliferation and Ffar2 expression beyond propionate-dependent pathways. Further mechanistic studies using actinomycin $\mathrm{D}$, an inhibitor of mRNA synthesis, revealed that CMTB regulates Ffar 2 expression at the transcription level independently of an activation of STAT3 (Supplementary Figure S2). Incubation of two human leukaemic cell lines (HL-60 and U937) in the presence of trichostatin A and doxorubicin revealed a similar association between cell proliferation and induction of Ffar2 expression (Supplementary Figure S3), allowing to extend the relevance of our finding beyond one mouse cell line.

We then sought to explore the impact of a modulation of Ffar2 expression on cancer cell proliferation in vivo. As overexpression of Ffar2 was previously shown to induce apoptosis in HEK293T cells (Shi et al, 2014), we rather investigated the impact of its knocking down. We used shRNA in a 13-day in vivo experiment. Transduction of $\mathrm{BaF}$ cells with a shRNA targeting FFAR2
A

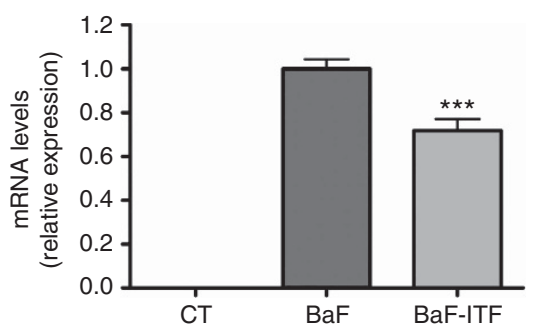

B

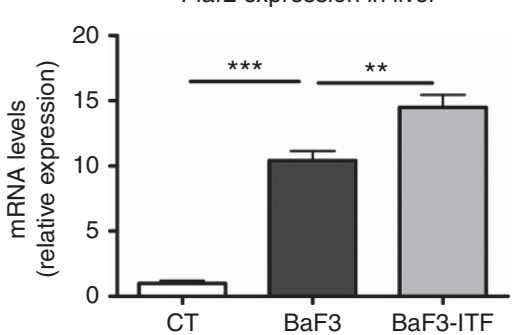

C

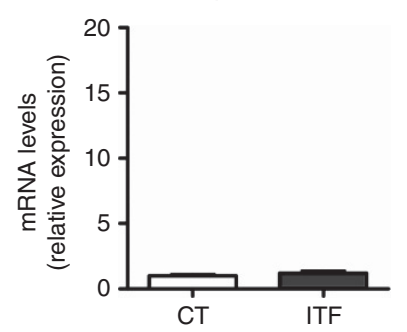

Figure 1. Reduced cell proliferation is associated with increased Ffar2 expression in vivo. Bcr-Abl and Ffar2 expression in the liver of BaFtransplanted mice with or without ITF (A, B). Ffar2 expression in the liver of mice with or without ITF (C). CT: control mice. ${ }^{\star \star} P<0.01$ and $\star \star \star P<0.001$ vs BaF, $n=8$. Figure 1A published in Bindels et al, 2012. 
A

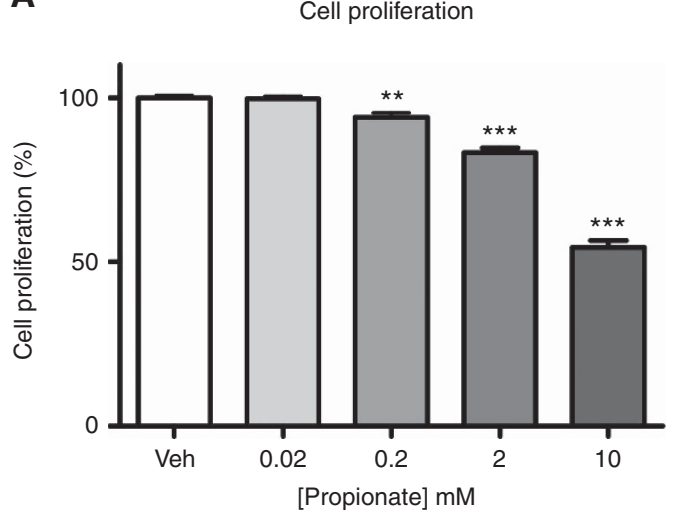

C

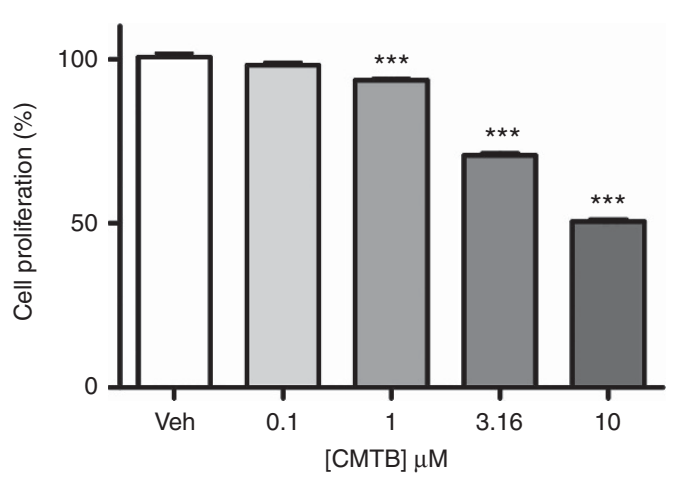

E

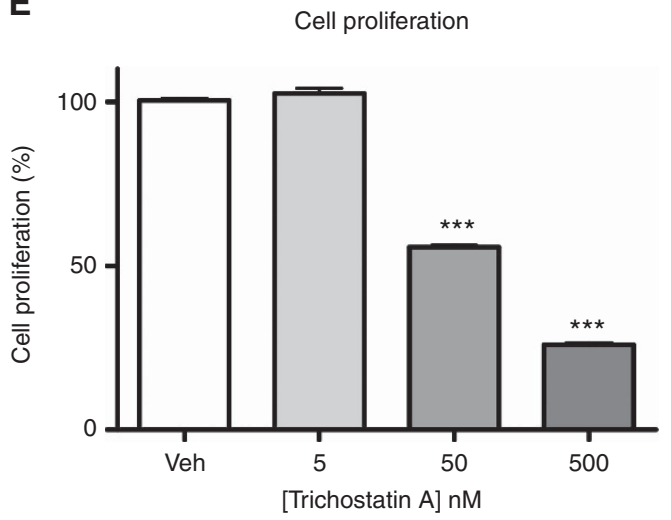

G

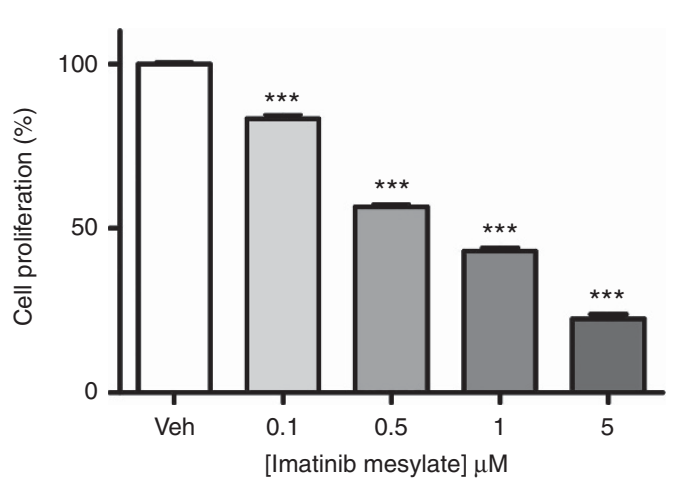

B

Ffar2 expression

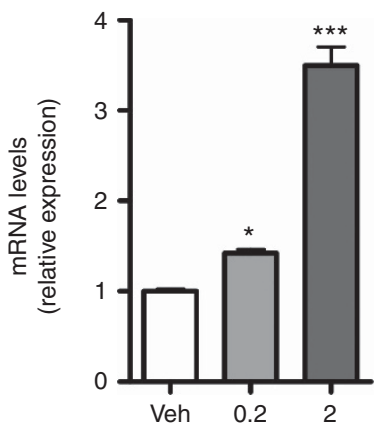

[Propionate] $\mathrm{mM}$

D

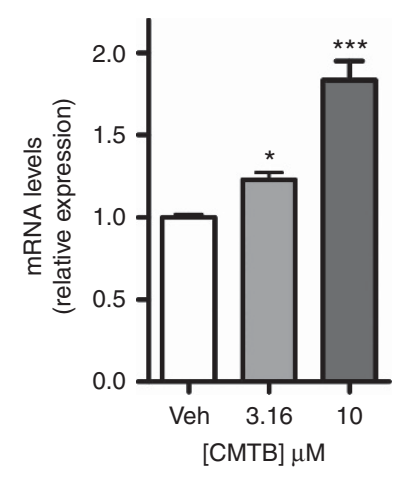

$\mathbf{F}$

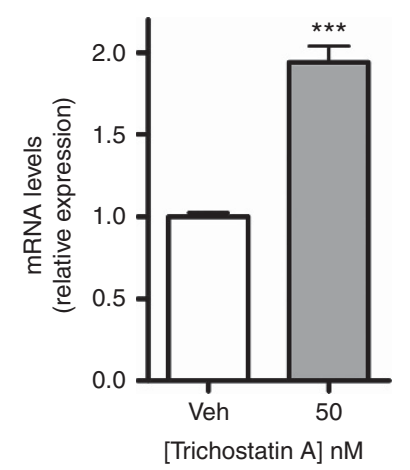

H

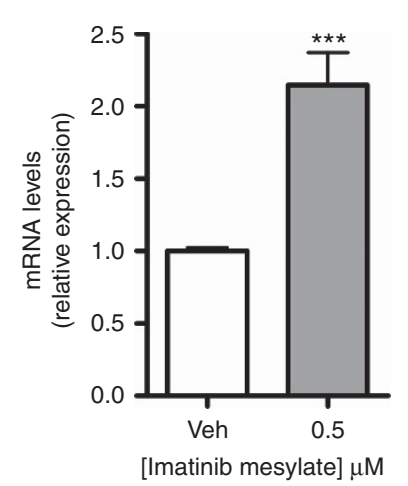

Figure 2. Reduced cell proliferation is associated with increased Ffar2 expression in vitro. Cell proliferation and Ffar2 expression after $24 \mathrm{~h}$ incubation in presence of propionate $(\mathbf{A}, \mathbf{B}), \operatorname{CMTB}(\mathbf{C}, \mathbf{D})$, trichostatin $\mathbf{A}(\mathbf{E}, \mathbf{F})$ and imatinib mesylate $(\mathbf{G}, \mathbf{H})$. Results of three independent experiments performed at least in triplicate are shown. ${ }^{\star} P<0.05,{ }^{\star \star} P<0.01,{ }^{\star \star \star} P<0.001$ vs vehicle (Veh). 
(shFFAR2) reduced target expression by 90\% (Figure 3A) and increased cell proliferation (Figure $3 \mathrm{~B}$ ). After in vivo transplantation, compared to BaF-shCT cells, BaF-shFFAR2 cells were found at higher levels in the blood, spleen, lungs, subcutaneous adipose tissue, but not in the liver (Figure 3C-G). In the liver, Ffar2 expression was increased in mice transplanted with BaF-shCT, but not in mice transplanted with BaF-shFFAR2 cells (Figure $3 \mathrm{H}$ ), further indicating that the increased hepatic expression of Ffar2 observed in our model is due to the expression of Ffar2 in BaF cells and not to changes of the expression of Ffar 2 in hepatic cells.

\section{DISCUSSION}

Our data formally establish that reduced leukaemic cell proliferation is associated with increased Ffar2 expression in vivo and in vitro. Furthermore, we show that knocking down Ffar2 expression directly promotes cancer cell spreading in vivo, at least in the serum, lungs, spleen and adipose tissue of mice. A relationship between cancer cell proliferation and expression of Ffar2 has been recently reported: loss of Ffar2 expression fosters colon carcinogenesis (Pan et al, 2016; Sivaprakasam et al, 2016) whereas overexpression of Ffar 2 decreases cell proliferation (Tang et al, 2011; Shi et al, 2014). However, the mechanism of action of
FFAR2 on cell growth remains unclear. We believe that the cellular model we generated here could contribute to the future elucidation of the underlying molecular pathways.

Regulation of murine Ffar2 expression is not well understood. Senga et al (2003) showed that murine Ffar2 expression in M1 leukaemia cells is associated with cell differentiation and is under the control of STAT3. Murine Ffar2 expression also positively correlates with the expression of differentiation factors in the adipose tissue (Dewulf et al, 2011). It was therefore tempting to link Ffar 2 expression to cell differentiation in BaF cells. However, mRNA expression of lymphocyte B differentiation markers (early $B$ cell factor 1 and CD19) in BaF cells was not detected after $24 \mathrm{~h}$ or $72 \mathrm{~h}$ of incubation in the presence of propionate $(10 \mathrm{~mm})$ or CMTB $(10 \mu \mathrm{M})$. In $\mathrm{BaF}$ cells, the increased murine Ffar2 expression occurred independently of STAT3 activation, suggesting the existence of cell type-dependent mechanisms involved in the regulation of Ffar2.

In the context of leukaemia treatment, our data revealed that ITF feeding to BaF-transplanted mice increases Ffar2 expression specifically in BaF cells residing in the liver. However, it remains to be determined whether the modulation of Ffar2 expression by ITF (likely through the production of propionate) constitutes one of the pathways by which ITF reduces leukaemic cell proliferation. Beside specific nutritional tools, pharmacological agents aiming at increasing the expression of Ffar2 in leukaemic cells could
A

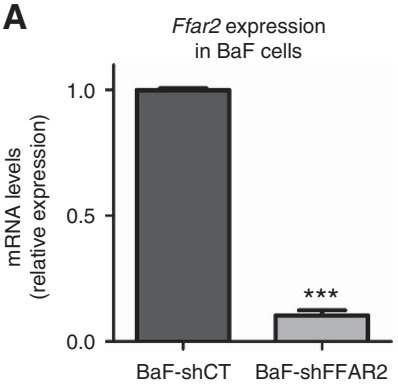

C

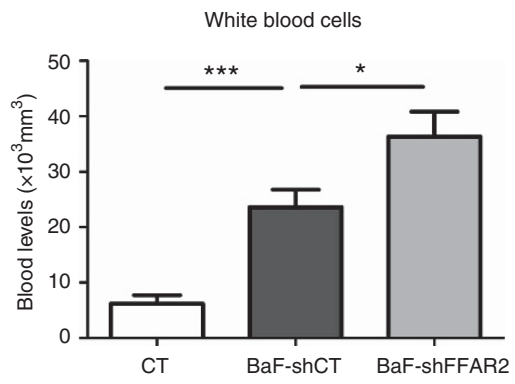

$\mathbf{F}$

Bcr-Abl expression in subcutaneous adipose tissue

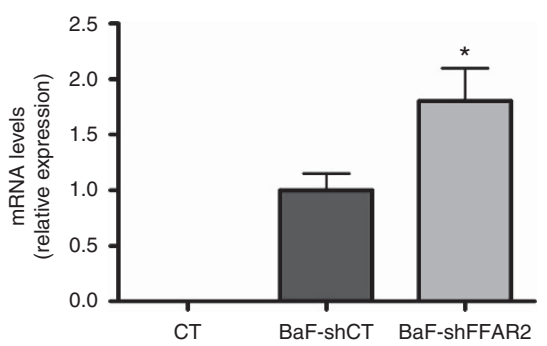

B

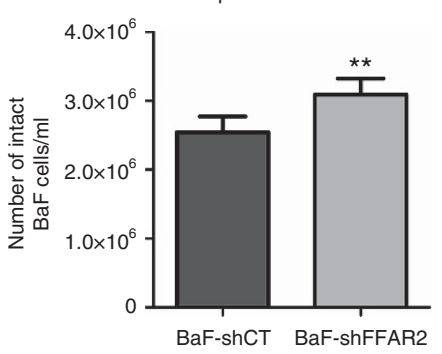

D

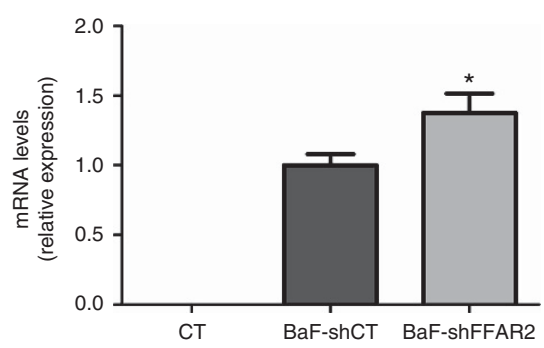

G

Bcr-Abl expression in liver

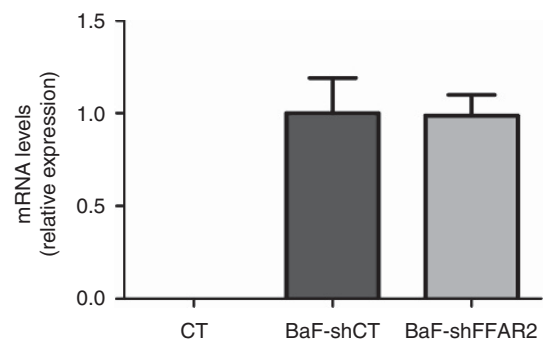

E

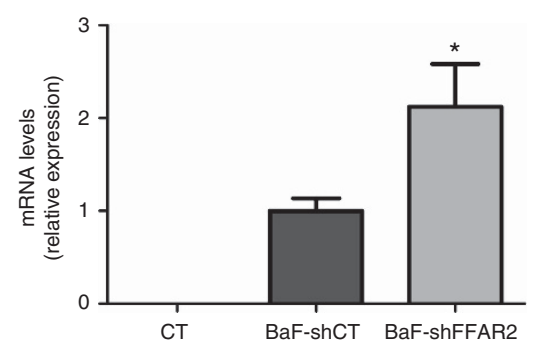

H

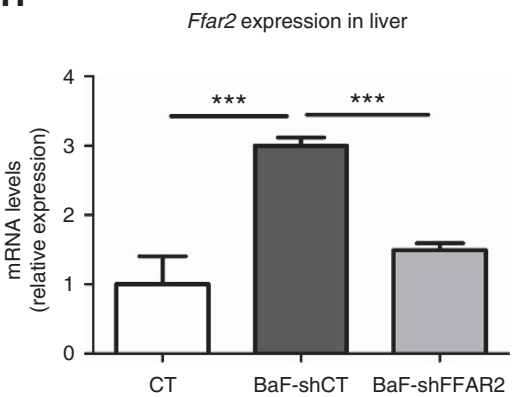

Figure 3. Decreasing Ffar2 expression increases leukaemic cell accumulation in vivo. Ffar2 expression in BaF cells in vitro (five independent experiments performed in triplicate) (A). Number of intact BaF cells per $\mathrm{ml} 48 \mathrm{~h}$ after incubation (four independent experiments) (B). White blood cell count (C). Bcr-Abl expression in spleen (D), lungs (E), subcutaneous adipose tissue (F) and liver (G). Ffar2 expression in liver (H). For $\mathbf{C}-\mathbf{H}$, $\mathrm{CT}$ : control mice $(n=4)$, BaF-shCT: mice transplanted with BaF cells transduced with a control shRNA $(n=8)$, BaF-shFFAR2: mice transplanted with BaF cells transduced with a shRNA targeting FFAR2 $(n=9)$. ${ }^{\star} P<0.05,{ }^{\star *} P<0.01,{ }^{\star \star \star} P<0.001$ vs BaF-shCT. Paired $t$-test for B. 
constitute an alternative approach to control leukaemic cell proliferation. Ang et al (2015) have recently shown that basal human Ffar2 expression is mainly under the control of $\mathrm{x}$-box binding protein 1 (XBP1). However, one should avoid to increase Ffar2 expression through activation of the XBP1 pathway, as inhibiting this pathway has been recently proposed as a promising therapeutic approach for acute myeloid leukaemia (Sun et al, 2016). Ang et al (2015) also suggested that several transcription factors may be involved in upregulating the gene under the appropriate external stimuli. Therefore, these alternative pathways need to be considered to increase human Ffar2 expression pharmacologically.

To conclude, our findings expand and clarify the current knowledge on the role and the therapeutic interest of FFAR2. Our data highlight that reduced cell proliferation is associated with increased Ffar2 expression in vitro and in vivo, and that modulation of Ffar2 expression regulates leukaemic cell growth in vitro and in vivo. Nutritional tools or pharmaceuticals targeting Ffar2 expression or activity might thus constitute an attractive therapeutic approach to tackle leukaemia progression in humans.

\section{ACKNOWLEDGEMENTS}

We thank Dr Julien Verrax for fruitful discussion, and Remi Selleslagh, Bouazza Es Saadi, Véronique Allaeys, Isabelle Blave and the team of Professor Gianello (Pôle de Chirurgie Expérimentale et Transplantation, UCL) for technical assistance. At the time of the study, MS was a Télévie PhD fellow, LBB and PEP Postdoctoral Researchers, PDC a Research Associate and PS a Senior Research Associate of the FRS-FNRS (Fond National de la Recherche Scientifique). NMD is recipient of FRS-FNRS grants. PDC is recipient of an ERC Starting Grant 2013 (European Research Council, Starting Grant 336452-ENIGMO), a Baillet Latour grant for medical research 2015 and is supported by the FRS-FNRS via FRFS-WELBIO under Grant number WELBIO-CR-2012S-02R. For this study, OF and PS were supported by Interuniversity Attraction Pole (IAP) grant \#UP7-03 from the Belgian Science Policy Office (Belspo) and by an Action de Recherche Concertée from the Communauté Française de Belgique (ARC 14/19-058).

\section{CONFLICT OF INTEREST}

The authors declare no conflict of interest.

\section{REFERENCES}

Ang Z, Er JZ, Ding JL (2015) The short-chain fatty acid receptor GPR43 is transcriptionally regulated by XBP1 in human monocytes. Sci Rep 5: 8134 .

Bindels LB, Dewulf EM, Delzenne NM (2013) GPR43/FFA2:

physiopathological relevance and therapeutic prospects. Trends Pharmacol Sci 34(4): 226-232.

Bindels LB, Neyrinck AM, Claus SP, Le Roy CI, Grangette C, Pot B, Martinez I, Walter J, Cani PD, Delzenne NM (2016) Synbiotic approach restores intestinal homeostasis and prolongs survival in leukaemic mice with cachexia. ISME J 10(6): 1456-1470.

Bindels LB, Porporato P, Dewulf EM, Verrax J, Neyrinck AM, Martin JC, Scott KP, Buc CP, Feron O, Muccioli GG, Sonveaux P, Cani PD, Delzenne NM (2012) Gut microbiota-derived propionate reduces cancer cell proliferation in the liver. Br J Cancer 107(8): 1337-1344.

Cacace A, Sboarina M, Vazeille T, Sonveaux P (2016) Glutamine activates STAT3 to control cancer cell proliferation independently of glutamine metabolism. Oncogene 36(15): 2074-2084.

Canfora EE, Jocken JW, Blaak EE (2015) Short-chain fatty acids in control of body weight and insulin sensitivity. Nat Rev Endocrinol 11(10): 577-591.

Charrier C, Bertrand P, Gesson JP, Roche J (2006) Synthesis of rigid trichostatin A analogs as HDAC inhibitors. Bioorg Med Chem Lett 16(20): 5339-5344.

Dewulf EM, Cani PD, Neyrinck AM, Possemiers S, Van HA, Muccioli GG, Deldicque L, Bindels LB, Pachikian BD, Sohet FM, Mignolet E, Francaux M, Larondelle Y, Delzenne NM (2011) Inulin-type fructans with prebiotic properties counteract GPR43 overexpression and PPARgammarelated adipogenesis in the white adipose tissue of high-fat diet-fed mice. $J$ Nutr Biochem 22(8): 712-722.

Druker BJ (2008) Translation of the Philadelphia chromosome into therapy for CML. Blood 112(13): 4808-4817.

Pan P, Skaer CW, Wang HT, Oshima K, Huang YW, Yu J, Zhang J, Yearsley M, Agle K, Drobyski W, Chen X, Wang LS (2016) Loss of free fatty acid receptor 2 enhances colonic adenoma development and reduces the chemopreventive effects of black raspberries in ApcMin/ + mice. Carcinogenesis 38(1): 86-93.

Senga T, Iwamoto S, Yoshida T, Yokota T, Adachi K, Azuma E, Hamaguchi M, Iwamoto T (2003) LSSIG is a novel murine leukocytespecific GPCR that is induced by the activation of STAT3. Blood 101(3): $1185-1187$.

Shi G, Sun C, Gu W, Yang M, Zhang X, Zhai N, Lu Y, Zhang Z, Shou P, Zhang Z, Ning G (2014) Free fatty acid receptor 2, a candidate target for type 1 diabetes, induces cell apoptosis through ERK signaling. $J \mathrm{Mol}$ Endocrinol 53(3): 367-380.

Sivaprakasam S, Gurav A, Paschall AV, Coe GL, Chaudhary K, Cai Y, Kolhe R, Martin P, Browning D, Huang L, Shi H, Sifuentes H, Vijay-Kumar M, Thompson SA, Munn DH, Mellor A, McGaha TL, Shiao P, Cutler CW, Liu K, Ganapathy V, Li H, Singh N (2016) An essential role of Ffar2 (Gpr43) in dietary fibre-mediated promotion of healthy composition of gut microbiota and suppression of intestinal carcinogenesis. Oncogenesis 5(6): e238.

Sun H, Lin DC, Guo X, Kharabi MB, Gery S, Cao Q, Alkan S, Ikezoe T, Akiba C, Paquette R, Chien W, Muller-Tidow C, Jing Y, Agelopoulos K, Muschen M, Koeffler HP (2016) Inhibition of IRE1alpha-driven prosurvival pathways is a promising therapeutic application in acute myeloid leukaemia. Oncotarget 7(14): 18736-18749.

Tang Y, Chen Y, Jiang H, Robbins GT, Nie D (2011) G-protein-coupled receptor for short-chain fatty acids suppresses colon cancer. Int $J$ Cancer 128(4): 847-856.

Wang Y, Jiao X, Kayser F, Liu J, Wang Z, Wanska M, Greenberg J, Weiszmann J, Ge H, Tian H, Wong S, Schwandner R, Lee T, Li Y (2010) The first synthetic agonists of FFA2: discovery and SAR of phenylacetamides as allosteric modulators. Bioorg Med Chem Lett 20(2): 493-498.

(c) (i) (2) This work is licensed under the Creative Commons (c) Attribution-Non-Commercial-Share Alike 4.0 International License. To view a copy of this license, visit http:// creativecommons.org/licenses/by-nc-sa/4.0/

(C) The Author(s) named above 2017

Supplementary Information accompanies this paper on British Journal of Cancer website (http://www.nature.com/bjc) 